\title{
DETERMINING MODES FOR THE 3D NAVIER-STOKES EQUATIONS
}

\author{
ALEXEY CHESKIDOV, MIMI DAI, AND LANDON KAVLIE
}

\begin{abstract}
We introduce a determining wavenumber for the forced 3D NavierStokes equations (NSE) defined for each individual solution. Even though this wavenumber blows up if the solution blows up, its time average is uniformly bounded for all solutions on the weak global attractor. The bound is compared to Kolmogorov's dissipation wavenumber and the Grashof constant.

KEY WORDS: Navier-Stokes equations, determining modes, global attractor.

CLASSIFICATION CODE: 35Q35, 37L30.
\end{abstract}

\section{INTRODUCTION}

The Navier-Stokes equations (NSE) on a torus, are given by

$$
\left\{\begin{array}{l}
u_{t}+(u \cdot \nabla) u-\nu \Delta u+\nabla p=f \\
\nabla \cdot u=0
\end{array}\right.
$$

where $u$ is the velocity, $p$ is the pressure, and $f$ is the external force. We assume that $f$ has zero mean, and consider zero mean solutions.

The dissipative nature of these equations is reflected in the existence of an absorbing ball in $L^{2}$. Moreover, in the two-dimensional case, there exists a compact global attractor which uniformly attracts all bounded subsets of $L^{2}$. This attracting set is, in fact, finite dimensional, as was proven by Foias and Temam in [17] (see also 11). The first result for the finite dimensionality of a two-dimensional fluid appeared in the work of Foias and Prodi 15, where they showed that high modes of a solution are controlled by low modes asymptotically as time goes to infinity. The number of these low modes, called determining modes, was estimated by Foias, Manley, Temam and Treve [14] and later improved by Jones and Titi 23]. See also [8, 12, 18, 20] and references therein for related results.

In three dimensions the situation is drastically different as the equations have thus far eluded a proof for the existence of classical solutions. Even so, the existence of a global attractor for weak solutions is known in a weak sense [19, 13], (see also 28. for a related notion of a trajectory attractor). This weak global attractor consists of points on complete bounded trajectories and attracts all bounded subsets of $L^{2}$ in the weak topology. However, it is not known whether the solutions on the attractor are regular, unless the attractor consists of a single fixed point. Neither is it known whether the attractor is compact or finite-dimensional. Similarly, the existence of a finite number of determining modes is not known in the three-dimensional case. Nevertheless, Constantin, Foias, Manley, and Temam [10] showed the existence of determining modes assuming that the $H^{1}$ norm of solutions is uniformly bounded.

The authors were partially supported by NSF Grant DMS-1108864 and DMS-1517583. 
The question whether the global attractor of the 3D NSE is bounded in $H^{1}$ is open and may very well require a resolution of the regularity problem. However, even assuming regularity, this would not immediately guarantee that the $H^{1}$ bound would depend only on the size of the force (a Grashof constant), and not on the shape of the force.

With no hope of getting a finite number of determining modes for the 3D NSE, one might ask whether this can be done in some average sense. Indeed, the Kolmogorov 41 phenomenological theory of turbulence [25] predicts that the number of degrees of freedom should be of order $\kappa_{\mathrm{d}}^{3}$, where $\kappa_{\mathrm{d}}$ is Kolmogorov's dissipation wavenumber. This number is often used as the resolution needed for direct numerical simulations, so one might ask an alternative question: What is the number of determining modes for a time discretization of the 3D NSE and how does it depend on the force and time step?

In this paper, without making any assumptions regarding regularity properties of solutions or bounds on the global attractor, we prove the existence of a timedependent determining wavenumber $\Lambda_{u}(t)$ defined for each individual solution $u$. We show that any weak solutions on the global attractor $u$ and $v$ that coincide below $\max \left\{\Lambda_{u}, \Lambda_{v}\right\}$ have to be identical. The wavenumber $\Lambda_{u}(t)$ blows up if and only if the solution $u(t)$ blows up. Nevertheless, the time average of this wavenumber is uniformly bounded on the global attractor, which we estimate in terms of the Kolmogorov dissipation number and Grashof constant.

To begin, let $u$ be a weak solution of the 3D Navier-Stokes equations. For $r \in(2,3)$ we define a local determining wavenumber

$$
\Lambda_{u, r}(t):=\min \left\{\lambda_{q}: \lambda_{p}^{-1+\frac{3}{r}}\left\|u_{p}\right\|_{L^{r}}<c_{r} \nu, \forall p>q \text { and } \lambda_{q}^{-1}\left\|u_{\leqslant q}\right\|_{L^{\infty}}<c_{r} \nu, q \in \mathbb{N}\right\},
$$

where $c_{r}$ is an adimensional constant that depends only on $r$ (it will be choosen in Section (3). Here $\lambda_{q}=\frac{2^{q}}{L}, L$ is the size of the torus, $u_{q}=\Delta_{q} u$ is the LittlewoodPaley projection of $u$, and $u_{\leqslant q}=\sum_{-1 \leqslant p \leqslant q} u_{p}$ (see Section 2). Notice that $\Lambda_{u, r}(t)$ might not be finite as we adopt a convention that $\min \varnothing=\infty$.

Thanks to Bernstein's inequality, we have

$$
\Lambda_{u, r} \geqslant \Lambda_{u}^{\mathrm{dis}}:=\min \left\{\lambda_{q}: \lambda_{p}^{-1}\left\|u_{p}\right\|_{L^{\infty}}<c_{0} \nu, \forall p>q, q \in \mathbb{N}\right\},
$$

which is a local dissipation wavenumber introduced by Cheskidov and Shvydkoy in 6]. It defines a dissipation range where a local Reynolds number corresponding to high frequencies is small, i.e.,

$$
\mathcal{R}_{q}^{h}:=\frac{l_{q}\left\|u_{q}\right\|_{L^{\infty}}}{\nu}<c_{0}, \quad \forall \lambda_{q}>\Lambda_{u}^{\mathrm{dis}},
$$

where $l_{q}=\lambda_{q}^{-1}$. The dominance of the dissipation term above $\Lambda_{u}^{\text {dis }}$ is reflected in improved Beale-Kato-Majda and Prodi-Serrin criteria where $u$ is replaced with its projection on modes below $\Lambda_{u}^{\text {dis }}$ (see [6]). In particular, $\Lambda_{u}^{\text {dis }}$ (and hence $\Lambda_{u, r}$ ) blows up if and only if the solution $u$ blows up. The determining wavenumber $\Lambda_{u, r}$ imposes tougher condition on high modes, as well as requires a control on low modes via the low frequency Reynolds number

$$
\mathcal{R}_{q}^{l}:=\frac{l_{q}\left\|u_{\leqslant q}\right\|_{L^{\infty}}}{\nu}<c_{r}, \quad \lambda_{q}=\Lambda_{u, r} .
$$

It is also worth mentioning that a similar determining wavenumber is used in [4] to prove the existence of a finite number of determining modes for the surface quasi-geostrophic equation equation in critical and subcritical cases. Even though 
the determining wavenumber enjoys uniform bounds in those cases, it still proved useful to start with a time dependent wavenumber defined based on the structure of the equation only, and then study its dependence on the force using available bounds for the global attractor.

We prove the following.

Theorem 1.1. Let $u(t)$ and $v(t)$ be weak solutions of the $3 D$ Navier-Stokes equations. Let $\Lambda(t):=\max \left\{\Lambda_{u, r}(t), \Lambda_{v, r}(t)\right\}$ for some $r \in(2,3)$. Let $Q(t)$ be such that $\Lambda(t)=\lambda_{Q(t)}$. If

$$
u(t)_{\leqslant Q(t)}=v(t)_{\leqslant Q(t)}, \quad \forall t>0,
$$

then

$$
\lim _{t \rightarrow \infty}\|u(t)-v(t)\|_{L^{2}}=0
$$

The proof of this theorem, given in Section 3. also implies the following results:

Theorem 1.2. If $u(t)$ and $v(t)$ are two Leray-Hopf solutions on the weak global attractor $\mathcal{A}$ such that

$$
u(t)_{\leqslant Q(t)}=v(t)_{\leqslant Q(t)}, \quad \forall t<0,
$$

where $Q$ is given in Theorem 1.1, then

$$
u(t)=v(t), \quad \forall t \leqslant 0 .
$$

This establishes the existence of determining modes for the 3D Navier-Stokes equations. It is worthwhile to note that the determining wavenumber $\Lambda_{u, r}$ depends on time and may not be bounded. Actually, it is bounded if and only if $u$ is regular. However, the average determining wavenumber $\langle\Lambda\rangle=\frac{1}{T} \int_{t}^{t+T} \Lambda_{u, r}(\tau) d \tau$ always enjoys a uniform bound. Indeed, we establish the following pointwise bound:

$$
\Lambda_{u, r}(t) \lesssim_{r} \frac{\|\nabla u(t)\|_{L^{2}}^{2}}{\nu^{2}}
$$

We direct the readers to Section 2 for the $\lesssim_{r}$ notation. Note that this automatically provides a finite number of determining modes and recovers the results by Constantin, Foias, Manley, and Temam [10] in the case where $\|\nabla u(t)\|_{L^{2}}^{2}$ is bounded on the global attractor, which is known for small forces (laminar regimes). On the other hand, (1.4) holds in general for arbitrary forces and implies that $\langle\Lambda\rangle$ is uniformly bounded for all Leray-Hopf solutions on the global attractor, i.e., complete bounded trajectories. However, the bound (1.4) is sharp only in the case of extreme intermittency, where on average there is only one eddy at each dyadic scale. To make a connection with Kolmogorov's turbulence theory [25, 21] we have to define an intermittency dimension and analyze $\langle\Lambda\rangle$ in various intermittency regimes.

In Section 4 and in Section 5 we further examine $\langle\Lambda\rangle$, comparing it to Kolmogorov's dissipation wavenumber as well as the Grashof constant, defined as

$$
\kappa_{\mathrm{d}}:=\left(\frac{\varepsilon}{\nu^{3}}\right)^{\frac{1}{d+1}}, \quad G:=\frac{\|f\|_{H^{-1}}}{\nu^{2} \lambda_{0}^{1 / 2}}, \quad \varepsilon:=\lambda_{0}^{d} \nu\left\langle\|\nabla u\|_{L^{2}}^{2}\right\rangle,
$$

where $d \in[0,3]$ is the intermittency dimension. This parameter is defined in Section 4 in terms of the level of saturation of Bernstein's inequality. The case $d=3$, 
where there is no intermittency and eddies occupy the whole region, corresponds to Kolmogorov's regime. In this case the bounds read

$$
\langle\Lambda\rangle \lesssim \delta \kappa_{d}^{2+\delta} \lambda_{0}^{-1-\delta} \lesssim \delta \lambda_{0}\left(\frac{G^{2}}{T \nu^{2} \lambda_{0}^{2}}+G^{2}\right)^{\frac{1}{2}+\delta}, \quad d=3,
$$

where $\delta$ can be arbitrary small when $r$ is chosen close to 3 . On the other hand, in the case of extreme intermittency, the bounds become

$$
\langle\Lambda\rangle \lesssim \kappa_{d} \lesssim \frac{G^{2}}{T \nu^{2} \lambda_{0}}+\lambda_{0} G^{2}, \quad d=0 .
$$

Finally, in Section 6 we show that if one of the weak solutions in Theorems 1.1 1.2 satisfies the energy equality, then $\Lambda$ can be defined in terms of that solution only. The energy equality holds for regular solutions, such as steady states, or

solutions belonging to Onsager's space $L^{3}\left(0, \infty ; B_{3, \infty}^{1 / 3}\right)$ (see [3] where the proof can be applied to the viscous case, where the space $B_{3, c_{0}}^{1 / 3}$ can be replaced with $B_{3, \infty}^{1 / 3}$ ).

\section{Preliminaries}

2.1. Notation. We denote by $A \lesssim B$ an estimate of the form $A \leqslant C B$ with some absolute constant $C$, by $A \sim B$ an estimate of the form $C_{1} B \leqslant A \leqslant C_{2} B$ with some absolute constants $C_{1}, C_{2}$, and by $A \lesssim_{r} B$ an estimate of the form $A \leqslant C_{r} B$ with some adimentional constant $C_{r}$ that depends only on the parameter $r . L^{p}$ and $H^{s}$ stand for Lebesgue and $L^{2}$-based Sobolev spaces respectively. To simplify the notations we will write $\|\cdot\|_{p}=\|\cdot\|_{L^{p}}$, and use $(\cdot, \cdot)$ for the $L^{2}$-inner product.

\subsection{Definition of solutions.}

Definition 2.1. A Leray-Hopf solution of (1.1) on $[0, T]$ is a function $u:[0, T] \rightarrow$ $L^{2}\left(\mathbb{R}^{3}\right)$ in the class

$$
u \in C_{\mathrm{w}}\left([0, T] ; L^{2}\left(\mathbb{T}^{3}\right)\right) \cap L_{\mathrm{loc}}^{2}\left([0, T] ; H^{1}\left(\mathbb{T}^{3}\right)\right),
$$

satisfying $\nabla \cdot u=0$ in the sense of distributions,

$$
(u(t), \varphi(t))+\int_{0}^{t}\left[-\left(u, \partial_{t} \varphi\right)+\nu(\nabla u, \nabla \varphi)+(u \cdot \nabla u, \varphi)+(f, \varphi)\right] d s=\left(u_{0}, \varphi(0)\right),
$$

for all $t \in[0, T]$ and all test functions $\varphi \in C^{\infty}\left([0, T] \times \mathbb{T}^{3}\right)$ with $\nabla \cdot \varphi=0$, and satisfying the energy inequality

$$
\frac{1}{2}\|u(t)\|_{2}^{2} \leqslant \frac{1}{2}\left\|u\left(t_{0}\right)\right\|_{2}^{2}+\int_{t_{0}}^{t}\left[-\|\nabla u(s)\|_{2}^{2}+(f, u)\right] d s,
$$

for almost all $t_{0} \in(0, T)$ and all $t \in\left[t_{0}, T\right]$.

Even though the global existence of Leray-Hopf solutions (for all $T>0$ ) is known (first proved by Leray in 1934 in the case of the whole space [26]), the uniqueness remains an open problem.

By a standard approximation argument, the function $\varphi$ in the definition of weak solution can be taken to be only weakly Lipschitz in time (see [27]). This will allow us to use Littlewood-Paley projections of $u(t)$ as test functions. 
2.3. Littlewood-Paley decomposition. The techniques presented in this paper rely strongly on the Littlewood-Paley decomposition. Thus we here recall the Littlewood-Paley decomposition theory briefly. For a more detailed description on this theory we refer the readers to the books by Bahouri, Chemin and Danchin [1] and Grafakos 22.

We denote $\lambda_{q}=\frac{2^{q}}{L}$ for integers $q$. A nonnegative radial function $\chi \in C_{0}^{\infty}\left(\mathbb{R}^{3}\right)$ is chosen such that

$$
\chi(\xi):= \begin{cases}1, & \text { for }|\xi| \leqslant \frac{3}{4} \\ 0, & \text { for }|\xi| \geqslant 1 .\end{cases}
$$

Let

$$
\varphi(\xi):=\chi(\xi / 2)-\chi(\xi)
$$

and

$$
\varphi_{q}(\xi):=\left\{\begin{array}{l}
\varphi\left(2^{-q} \xi\right) \quad \text { for } q \geqslant 0, \\
\chi(\xi) \quad \text { for } q=-1,
\end{array}\right.
$$

so that the sequence of $\varphi_{q}$ forms a dyadic partition of unity. Given a tempered distribution vector field $u$ on $\mathbb{T}^{3}=[0, L]^{3}$ and $q \geqslant-1$, an integer, the $q$ th LittlewoodPaley projection of $u$ is given by

$$
u_{q}(x):=\Delta_{q} u(x):=\sum_{k \in \mathbb{Z}^{3}} \widehat{u}(k) \varphi_{q}(k) e^{i \frac{2 \pi}{L} k \cdot x},
$$

where $\widehat{u}(k)$ is the $k$ th Fourier coefficient of $u$. Note that $u_{-1}=\widehat{u}(0)$. Then

$$
u=\sum_{q=-1}^{\infty} u_{q}
$$

in the distributional sense. Note that

$$
\|u\|_{H^{s}} \sim\left(\sum_{q=-1}^{\infty} \lambda_{q}^{2 s}\left\|u_{q}\right\|_{2}^{2}\right)^{1 / 2},
$$

for each $u \in H^{s}$ and $s \in \mathbb{R}$. To simplify the notations, we denote

$$
u_{\leqslant Q}:=\sum_{q=-1}^{Q} u_{q}=\sum_{k \in \mathbb{Z}^{3}} \widehat{u}(k) \chi\left(2^{-Q-1} k\right) e^{i \frac{2 \pi}{L} k \cdot x} .
$$

Next we prove the following property for the dyadic blocks.

Lemma 2.2. For any $Q \geqslant-1$, the identity

$$
u_{Q}=\left(\Delta_{Q}+\Delta_{Q+1}\right) u_{\leqslant Q}
$$

holds.

Proof: We denote

$$
\psi_{Q}:=\sum_{-1 \leqslant q \leqslant Q} \varphi_{q} .
$$

It follows from (2.6) and (2.7) that $\psi_{Q}(\xi)=\chi\left(2^{-Q-1} \xi\right)$. By (2.8), in order to prove the lemma, it is sufficient to show that

$$
\varphi_{Q}=\psi_{Q}\left(\varphi_{Q}+\varphi_{Q+1}\right) \text {. }
$$


Indeed, we have from (2.6) and (2.7),

$$
\varphi_{Q}(\xi)+\varphi_{Q+1}(\xi)=\chi\left(2^{-Q-2} \xi\right)-\chi\left(2^{-Q} \xi\right) .
$$

Thus,

$$
\psi_{Q}(\xi)\left[\varphi_{Q}(\xi)+\varphi_{Q+1}(\xi)\right]=\chi\left(2^{-Q-1} \xi\right)\left[\chi\left(2^{-Q-2} \xi\right)-\chi\left(2^{-Q} \xi\right)\right] .
$$

It then follows from the facts

$$
\chi\left(2^{-Q-1} \xi\right) \chi\left(2^{-Q-2} \xi\right)=\chi\left(2^{-Q-1} \xi\right), \quad \chi\left(2^{-Q-1} \xi\right) \chi\left(2^{-Q} \xi\right)=\chi\left(2^{-Q} \xi\right),
$$

that

$$
\psi_{Q}(\xi)\left[\varphi_{Q}(\xi)+\varphi_{Q+1}(\xi)\right]=\chi\left(2^{-Q-1} \xi\right)-\chi\left(2^{-Q} \xi\right)=\varphi_{Q}(\xi),
$$

which completes the proof.

2.4. Bony's paraproduct, Littlewood-Paley theorem, and inequalities. Here we state some useful properties for the dyadic blocks of the Littlewood-Paley decomposition and some inequalities that will be used often through the paper.

We start with Bony's paraproduct formula. First, note that

$$
u \cdot \nabla v=\sum_{p} u_{\leqslant p-2} \cdot \nabla v_{p}+\sum_{p} u_{p} \cdot \nabla v_{\leqslant p-2}+\sum_{p} \sum_{\left|p-p^{\prime}\right| \leqslant 1} u_{p} \cdot \nabla v_{p^{\prime}} .
$$

Due to (2.6) we have $\varphi(\xi)=0$ when $|\xi| \leqslant 3 / 4$ or $|\xi| \geqslant 2$, and hence

$$
\left(f_{q} g_{\leqslant q-2}\right)_{\geqslant q+2} \equiv 0, \quad\left(f_{q} g_{\leqslant q-2}\right)_{\leqslant q-3} \equiv 0, \quad\left(f_{q} g_{q+1}\right)_{\geqslant q+3} \equiv 0,
$$

for tempered distributions $f$ and $g$. Therefore,

$$
\begin{aligned}
\Delta_{q}(u \cdot \nabla v)= & \sum_{q-1 \leqslant p \leqslant q+2} \Delta_{q}\left(u_{\leqslant p-2} \cdot \nabla v_{p}\right)+\sum_{q-1 \leqslant p \leqslant q+2} \Delta_{q}\left(u_{p} \cdot \nabla v_{\leqslant p-2}\right) \\
& +\sum_{p \geqslant q-2} \sum_{\substack{\left|p-p^{\prime}\right| \leqslant 1 \\
p^{\prime} \geqslant q-2}} \Delta_{q}\left(u_{p} \cdot \nabla v_{p^{\prime}}\right) .
\end{aligned}
$$

It is usually sufficient to use a weaker form of this formula:

$$
\begin{aligned}
\Delta_{q}(u \cdot \nabla v)= & \sum_{|q-p| \leqslant 2} \Delta_{q}\left(u_{\leqslant p-2} \cdot \nabla v_{p}\right)+\sum_{|q-p| \leqslant 2} \Delta_{q}\left(u_{p} \cdot \nabla v_{\leqslant p-2}\right) \\
& +\sum_{p \geqslant q-2} \Delta_{q}\left(\tilde{u}_{p} \cdot \nabla v_{p}\right),
\end{aligned}
$$

where $\tilde{u}_{p}:=u_{p-1}+u_{p}+u_{p+1}$.

Finally, we will take advantage of the Littlewood-Paley Theorem, which yields the following bounds on the Lebesgue norms:

$$
\left(\sum_{q}\left\|u_{q}\right\|_{r}^{r}\right)^{\frac{1}{r}} \lesssim\|u\|_{r} \lesssim\left(\sum_{q}\left\|u_{q}\right\|_{2}^{2}\right)^{\frac{1}{2}}, \quad r \geqslant 2 .
$$

We recall Bernstein's inequality.

Lemma 2.3. Let $n$ be the spacial dimension and $r \geqslant s \geqslant 1$. Then for all tempered distributions $u$,

$$
\left\|u_{q}\right\|_{r} \lesssim \lambda_{q}^{n\left(\frac{1}{s}-\frac{1}{r}\right)}\left\|u_{q}\right\|_{s} .
$$

We will also use Jensen's inequality stated bellow. 
Lemma 2.4. Let $\varphi: I \rightarrow \mathbb{R}$ be a convex function on an interval $I$. For any points $x_{1}, x_{2}, \ldots, x_{n} \in I$ and weights $\theta_{1}, \theta_{2}, \ldots, \theta_{n} \in(0,1)$, the inequality

$$
\varphi\left(\theta_{1} x_{1}+\theta_{2} x_{2}+\cdots+\theta_{n} x_{n}\right) \leqslant \theta_{1} \varphi\left(x_{1}\right)+\theta_{2} \varphi\left(x_{2}\right)+\cdots+\theta_{n} \varphi\left(x_{n}\right) .
$$

holds.

\section{Proof of Theorems 1.1 and 1.2}

Let $w:=u-v$, which satisfies the equation

$$
w_{t}+u \cdot \nabla w+w \cdot \nabla v=-\nabla \pi+\nu \Delta w
$$

in the distribution sense (see Definition 2.1). By our assumption, $w_{\leqslant Q(t)}(t) \equiv 0$.

Using $\Delta_{q}^{2} w$ as a test function for (3.12) and adding up for all $q \geqslant-1$ yields

$$
\begin{aligned}
& \frac{1}{2}\|w(t)\|_{2}^{2}-\frac{1}{2}\left\|w\left(t_{0}\right)\right\|_{2}^{2}+\nu \int_{t_{0}}^{t}\|\nabla w\|_{2}^{2} d \tau \\
\leqslant & \int_{t_{0}}^{t} \sum_{q \geqslant-1}\left|\int_{\mathbb{T}^{3}} \Delta_{q}(w \cdot \nabla v) w_{q} d x\right| d \tau+\int_{t_{0}}^{t} \sum_{q \geqslant-1}\left|\int_{\mathbb{T}^{3}} \Delta_{q}(u \cdot \nabla w) w_{q} d x\right| d \tau, \\
= & \int_{t_{0}}^{t} I_{1} d \tau+\int_{t_{0}}^{t} I_{2} d \tau .
\end{aligned}
$$

Using Bony's paraproduct mentioned in Subsection 2.4 and the triangle inequality, $I_{1}$ is decomposed as

$$
\begin{aligned}
I_{1} \leqslant & \sum_{q \geqslant-1} \sum_{|q-p| \leqslant 2}\left|\int_{\mathbb{T}^{3}} \Delta_{q}\left(w_{\leqslant p-2} \cdot \nabla v_{p}\right) w_{q} d x\right| \\
& +\sum_{q \geqslant-1} \sum_{|q-p| \leqslant 2}\left|\int_{\mathbb{T}^{3}} \Delta_{q}\left(w_{p} \cdot \nabla v_{\leqslant p-2}\right) w_{q} d x\right| \\
& +\sum_{q \geqslant-1} \sum_{p \geqslant q-2}\left|\int_{\mathbb{T}^{3}} \Delta_{q}\left(\tilde{w}_{p} \cdot \nabla v_{p}\right) w_{q} d x\right| \\
=: & I_{11}+I_{12}+I_{13} .
\end{aligned}
$$

These terms are estimated as follows. Recall that $w_{\leqslant Q(t)} \equiv 0$. Then using Hölder's inequality we obtain

$$
\begin{aligned}
I_{11} & \leqslant \sum_{p \geqslant Q+3} \sum_{|q-p| \leqslant 2} \int_{\mathbb{T}^{3}}\left|\Delta_{q}\left(w_{\leqslant p-2} \cdot \nabla v_{p}\right) w_{q}\right| d x \\
& \lesssim \sum_{p \geqslant Q+3} \sum_{|q-p| \leqslant 2}\left\|w_{\leqslant p-2}\right\|_{\frac{2 r}{r-2}} \lambda_{p}\left\|v_{p}\right\|_{r}\left\|w_{q}\right\|_{2} .
\end{aligned}
$$


Now using definition of $\Lambda_{v, r}$, Bernstein's inequality (2.10), Young's inequality and Jensen's inequality (2.11), we get

$$
\begin{aligned}
I_{11} & \lesssim c_{r} \nu \sum_{p \geqslant Q+3} \sum_{|q-p| \leqslant 2} \lambda_{p}^{2-\frac{3}{r}}\left\|w_{q}\right\|_{2} \sum_{p^{\prime} \leqslant p-2}\left\|w_{p^{\prime}}\right\|_{\frac{2 r}{r-2}} \\
& \lesssim c_{r} \nu \sum_{q \geqslant Q+1} \lambda_{q}\left\|w_{q}\right\|_{2} \sum_{p^{\prime} \leqslant q} \lambda_{p^{\prime}}\left\|w_{p^{\prime}}\right\|_{2} \lambda_{q-\frac{p^{\prime}}{r}}^{1-\frac{3}{2}} \\
& \lesssim c_{r} \nu \sum_{q \geqslant Q+1} \lambda_{q}^{2}\left\|w_{q}\right\|_{2}^{2}+c_{r} \nu \sum_{q \geqslant Q+1}\left(\sum_{p^{\prime} \leqslant q} \lambda_{p^{\prime}}\left\|w_{p^{\prime}}\right\|_{2} \lambda_{q-p^{\prime}}^{1-\frac{3}{r}}\right)^{2} \\
& \lesssim c_{r} \nu \sum_{q \geqslant Q+1} \lambda_{q}^{2}\left\|w_{q}\right\|_{2}^{2}+c_{r} \nu \sum_{q \geqslant Q+1} \sum_{p^{\prime} \leqslant q} \lambda_{p^{\prime}}^{2}\left\|w_{p^{\prime}}\right\|_{2}^{2} \lambda_{q-\frac{p^{\prime}}{r}}^{1-\frac{3}{r}} \\
& \lesssim c_{r} \nu \sum_{q \geqslant Q+1} \lambda_{q}^{2}\left\|w_{q}\right\|_{2}^{2}+c_{r} \nu \sum_{-1 \leqslant p^{\prime} \leqslant Q} \lambda_{p^{\prime}}^{2}\left\|w_{p^{\prime}}\right\|_{2}^{2} \sum_{q \geqslant Q} \lambda_{q-\frac{p^{\prime}}{r}}^{1-\frac{3}{p^{\prime}}}+c_{r} \nu \sum_{p^{\prime} \geqslant Q} \lambda_{p^{\prime}}^{2}\left\|w_{p^{\prime}}\right\|_{2}^{2} \sum_{q \geqslant p^{\prime}} \lambda_{q-\frac{p^{\prime}}{r}}^{1-\frac{3}{p^{\prime}}} \\
& \lesssim c_{r} \nu\|\nabla w\|_{2}^{2},
\end{aligned}
$$

where we used the fact that $r<3$.

Next, we have

$$
\begin{aligned}
I_{12} & \leqslant \sum_{p>Q} \sum_{|q-p| \leqslant 2} \int_{\mathbb{T}^{3}}\left|\Delta_{q}\left(w_{p} \cdot \nabla v_{\leqslant p-2}\right) w_{q}\right| d x \\
& \leqslant \sum_{p>Q} \sum_{|q-p| \leqslant 2} \int_{\mathbb{T}^{3}}\left|\Delta_{q}\left(w_{p} \cdot \nabla v_{\leqslant Q}\right) w_{q}\right| d x+\sum_{p^{\prime}>Q} \sum_{p \geqslant p^{\prime}+2} \sum_{|q-p| \leqslant 2} \int_{\mathbb{T}^{3}}\left|\Delta_{q}\left(w_{p} \cdot \nabla v_{p^{\prime}}\right) w_{q}\right| d x \\
& \lesssim\left\|\nabla v_{\leqslant Q}\right\|_{\infty} \sum_{q>Q} \sum_{|q-p| \leqslant 2}\left\|w_{p}\right\|_{2}\left\|w_{q}\right\|_{2}+\sum_{p^{\prime}>Q}\left\|\nabla v_{p^{\prime}}\right\|_{\infty} \sum_{p \geqslant p^{\prime}+2} \sum_{|q-p| \leqslant 2}\left\|w_{p}\right\|_{2}\left\|w_{q}\right\|_{2} \\
& \lesssim\left\|\nabla v_{\leqslant Q}\right\|_{\infty} \sum_{q>Q}\left\|w_{q}\right\|_{2}^{2}+\sum_{p^{\prime}>Q} \lambda_{p^{\prime}}^{1+\frac{3}{r}}\left\|v_{p^{\prime}}\right\|_{r} \sum_{q \geqslant p^{\prime}}\left\|w_{q}\right\|_{2}^{2} .
\end{aligned}
$$

Using the definition of $\Lambda_{v, r}$ and changing the order of summation, we obtain

$$
\begin{aligned}
I_{12} & \lesssim c_{r} \nu \sum_{q>Q} \lambda_{Q} \lambda_{q}\left\|w_{q}\right\|_{2}^{2}+c_{r} \nu \sum_{p^{\prime}>Q} \lambda_{p^{\prime}}^{2} \sum_{q \geqslant p^{\prime}}\left\|w_{q}\right\|_{2}^{2} \\
& \lesssim c_{r} \nu \sum_{q>Q} \lambda_{q}^{2}\left\|w_{q}\right\|_{2}^{2}+c_{r} \nu \sum_{q>Q} \lambda_{q}^{2}\left\|w_{q}\right\|_{2}^{2} \sum_{Q<p^{\prime} \leqslant q} \lambda_{q-p^{\prime}}^{-2} \\
& \lesssim c_{r} \nu\|\nabla w\|_{2}^{2},
\end{aligned}
$$

where $\sum_{Q<p^{\prime} \leqslant q} \lambda_{q-p^{\prime}}^{-2} \lesssim 1$ is used in the last inequality.

We will now estimate $I_{13}$. Recall that $w_{\leqslant Q} \equiv 0$ and hence $\tilde{w}_{\leqslant Q-1} \equiv 0$. Since $\Delta_{q}$ and $\nabla$ commute, integration by parts and changing the order of summation yield

$$
\begin{aligned}
I_{13} & =\sum_{q \geqslant-1} \sum_{p \geqslant q-2}\left|\int_{\mathbb{T}^{3}} \Delta_{q}\left(\tilde{w}_{p} \cdot \nabla v_{p}\right) \cdot w_{q} d x\right| \\
& =\sum_{q>Q} \sum_{p \geqslant q-2}\left|\int_{\mathbb{T}^{3}} \Delta_{q}\left(\tilde{w}_{p} \otimes v_{p}\right): \nabla w_{q} d x\right| \\
& =\sum_{p \geqslant Q} \sum_{Q<q \leqslant p+2}\left|\int_{\mathbb{T}^{3}} \Delta_{q}\left(\tilde{w}_{p} \otimes v_{p}\right): \nabla w_{q} d x\right| .
\end{aligned}
$$


Thanks to Hölder's inequality, we have

$$
\begin{aligned}
I_{13} & =\sum_{p>Q} \sum_{Q<q \leqslant p+2}\left|\int_{\mathbb{T}^{3}} \Delta_{q}\left(\tilde{w}_{p} \otimes v_{p}\right): \nabla w_{q} d x\right|+\sum_{Q<q \leqslant Q+2}\left|\int_{\mathbb{T}^{3}} \Delta_{q}\left(\tilde{w}_{Q} \otimes v_{Q}\right): \nabla w_{q} d x\right| \\
& \lesssim \sum_{p>Q}\left\|\tilde{w}_{p}\right\|_{2}\left\|v_{p}\right\|_{r} \sum_{Q<q \leqslant p+2} \lambda_{q}\left\|w_{q}\right\|_{\frac{2 r}{r-2}}+\lambda_{Q} \sum_{Q<q \leqslant Q+2}\left\|\tilde{w}_{Q}\right\|_{2}\left\|v_{Q}\right\|_{\infty}\left\|w_{q}\right\|_{2} .
\end{aligned}
$$

By Lemma 2.2 and definition of $\Lambda_{v, r}$,

$$
\left\|v_{Q}\right\|_{\infty}=\left\|\left(\Delta_{Q}+\Delta_{Q+1}\right) v_{\leqslant Q}\right\|_{\infty} \lesssim\left\|v_{\leqslant Q}\right\|_{\infty} \leqslant c_{r} \nu \lambda_{Q} .
$$

Hence, it follows

$$
\begin{aligned}
I_{13} & \lesssim c_{r} \nu \sum_{p>Q} \lambda_{p}^{1-\frac{3}{r}}\left\|\tilde{w}_{p}\right\|_{2} \sum_{Q<q \leqslant p+2} \lambda_{q}^{1+\frac{3}{r}}\left\|w_{q}\right\|_{2}+c_{r} \nu \lambda_{Q}^{2} \sum_{Q<q \leqslant Q+2}\left\|\tilde{w}_{Q}\right\|_{2}\left\|w_{q}\right\|_{2} \\
& \lesssim c_{r} \nu \sum_{p>Q-1} \lambda_{p}\left\|w_{p}\right\|_{2} \sum_{Q<q \leqslant p+2} \lambda_{q}\left\|w_{q}\right\|_{2} \lambda_{q-p}^{\frac{3}{r}}+c_{r} \nu \lambda_{Q}^{2} \sum_{Q<q \leqslant Q+2}\left\|w_{q}\right\|_{2}^{2} .
\end{aligned}
$$

Then we apply Young's inequality and Jensen's inequality (2.11) to deduce

$$
\begin{aligned}
& I_{13} \lesssim c_{r} \nu \sum_{p>Q-1} \lambda_{p}^{2}\left\|w_{p}\right\|_{2}^{2}+c_{r} \nu \sum_{p>Q-1}\left(\sum_{Q<q \leqslant p+2} \lambda_{q}\left\|w_{q}\right\|_{2} \lambda_{q-p}^{\frac{3}{r}}\right)^{2} \\
& +c_{r} \nu \lambda_{Q}^{2} \sum_{Q<q \leqslant Q+2}\left\|w_{q}\right\|_{2}^{2} \\
& \lesssim c_{r} \nu \sum_{p>Q-1} \lambda_{p}^{2}\left\|w_{p}\right\|_{2}^{2}+c_{r} \nu \sum_{p>Q-1} \sum_{Q<q \leqslant p+2} \lambda_{q}^{2}\left\|w_{q}\right\|_{2}^{2} \lambda_{q-p}^{\frac{3}{r}} \\
& +c_{r} \nu \lambda_{Q}^{2} \sum_{Q<q \leqslant Q+2}\left\|w_{q}\right\|_{2}^{2} \\
& \lesssim c_{r} \nu \sum_{p>Q-1} \lambda_{p}^{2}\left\|w_{p}\right\|_{2}^{2}+c_{r} \nu \sum_{q>Q} \lambda_{q}^{2}\left\|w_{q}\right\|_{2}^{2} \sum_{p \geqslant q-2} \lambda_{q-p}^{\frac{3}{r}} \\
& +c_{r} \nu \lambda_{Q}^{2} \sum_{Q<q \leqslant Q+2}\left\|w_{q}\right\|_{2}^{2} \\
& \lesssim c_{r} \nu\|\nabla w\|_{2}^{2}
\end{aligned}
$$

Combining the estimates above leads to

$$
I_{1} \lesssim c_{r} \nu\|\nabla w\|_{2}^{2}
$$

To estimate $I_{2}$, we start with the decomposition

$$
\begin{aligned}
I_{2} \leqslant & \sum_{q \geqslant-1} \sum_{|q-p| \leqslant 2}\left|\int_{\mathbb{T}^{3}} \Delta_{q}\left(u_{\leqslant p-2} \cdot \nabla w_{p}\right) w_{q} d x\right| \\
& +\sum_{q \geqslant-1} \sum_{|q-p| \leqslant 2}\left|\int_{\mathbb{T}^{3}} \Delta_{q}\left(u_{p} \cdot \nabla w_{\leqslant p-2}\right) w_{q} d x\right| \\
& +\sum_{q \geqslant-1} \sum_{p \geqslant q-2} \sum_{\left|p-p^{\prime}\right| \leqslant 1}\left|\int_{\mathbb{T}^{3}} \Delta_{q}\left(u_{p} \cdot \nabla w_{p^{\prime}}\right) w_{q} d x\right| \\
= & I_{21}+I_{22}+I_{23} .
\end{aligned}
$$


Then we estimate the terms as follows:

$$
\begin{aligned}
I_{21} & =\sum_{p>Q} \sum_{|q-p| \leqslant 2}\left|\int_{\mathbb{T}^{3}} \Delta_{q}\left(u_{\leqslant p-2} \cdot \nabla w_{p}\right) w_{q} d x\right| \\
& \leqslant \sum_{p>Q} \sum_{|q-p| \leqslant 2} \int_{\mathbb{T}^{3}}\left|\Delta_{q}\left(u_{\leqslant Q} \cdot \nabla w_{p}\right) w_{q}\right| d x+\sum_{p^{\prime}>Q} \sum_{p \geqslant p^{\prime}+2} \sum_{|q-p| \leqslant 2} \int_{\mathbb{T}^{3}}\left|\Delta_{q}\left(u_{p^{\prime}} \cdot \nabla w_{p}\right) w_{q}\right| d x \\
& \lesssim\left\|u_{\leqslant Q}\right\|_{\infty} \sum_{p>Q} \sum_{|q-p| \leqslant 2} \lambda_{p}\left\|w_{p}\right\|_{2}\left\|w_{q}\right\|_{2}+\sum_{p^{\prime}>Q}\left\|u_{p^{\prime}}\right\|_{\infty} \sum_{p \geqslant p^{\prime}+2} \sum_{|q-p| \leqslant 2} \lambda_{p}\left\|w_{p}\right\|_{2}\left\|w_{q}\right\|_{2} \\
& \lesssim\left\|u_{\leqslant Q}\right\|_{\infty} \sum_{q>Q} \lambda_{q}\left\|w_{q}\right\|_{2}^{2}+\sum_{p^{\prime}>Q} \lambda_{p^{\prime}}^{\frac{3}{r}}\left\|u_{p^{\prime}}\right\|_{r} \sum_{q \geqslant p^{\prime}} \lambda_{q}\left\|w_{q}\right\|_{2}^{2} .
\end{aligned}
$$

Using the definition of $\Lambda_{u, r}$ and changing the order of summation, we arrive at

$$
\begin{aligned}
I_{21} & \lesssim c_{r} \nu \sum_{q>Q} \lambda_{Q} \lambda_{q}\left\|w_{q}\right\|_{2}^{2}+c_{r} \nu \sum_{p^{\prime}>Q} \lambda_{p^{\prime}} \sum_{q \geqslant p^{\prime}} \lambda_{q}\left\|w_{q}\right\|_{2}^{2} \\
& \lesssim c_{r} \nu \sum_{q>Q} \lambda_{q}^{2}\left\|w_{q}\right\|_{2}^{2}+c_{r} \nu \sum_{q>Q} \lambda_{q}^{2}\left\|w_{q}\right\|_{2}^{2} \sum_{Q<p^{\prime} \leqslant q} \lambda_{q-p^{\prime}}^{-1} \\
& \lesssim c_{r} \nu\|\nabla w\|_{2}^{2},
\end{aligned}
$$

where we used $\sum_{Q<p^{\prime} \leqslant q} \lambda_{q-p^{\prime}}^{-1} \lesssim 1$ for the last inequality.

We proceed to estimating the second term. First, Hölder's inequality and Bernstein's inequality (2.10) yield

$$
\begin{aligned}
I_{22} & =\sum_{q>Q} \sum_{|q-p| \leqslant 2}\left|\int_{\mathbb{T}^{3}} \Delta_{q}\left(u_{p} \cdot \nabla w_{\leqslant p-2}\right) w_{q} d x\right| \\
& \lesssim \sum_{q>Q} \sum_{|q-p| \leqslant 2}\left\|u_{p}\right\|_{r}\left\|w_{q}\right\|_{2} \sum_{p^{\prime} \leqslant q-2} \lambda_{p^{\prime}}\left\|w_{p^{\prime}}\right\|_{\frac{2 r}{r-2}} \\
& \lesssim c_{r} \nu \sum_{q>Q} \lambda_{q}^{1-\frac{3}{r}}\left\|w_{q}\right\|_{2} \sum_{p^{\prime} \leqslant q-2} \lambda_{p^{\prime}}^{1+\frac{3}{r}}\left\|w_{p^{\prime}}\right\|_{2} \\
& \lesssim c_{r} \nu \sum_{q>Q} \lambda_{q}\left\|w_{q}\right\|_{2} \sum_{p^{\prime} \leqslant q-2} \lambda_{p^{\prime}}\left\|w_{p^{\prime}}\right\|_{2} \lambda_{q-p^{\prime}}^{-\frac{3}{r}} .
\end{aligned}
$$

We continue by using Young's inequality, Jensen's inequality (2.11), and changing the order of summation to obtain

$$
\begin{aligned}
I_{22} & \lesssim c_{r} \nu \sum_{q>Q} \lambda_{q}^{2}\left\|w_{q}\right\|_{2}^{2}+c_{r} \nu \sum_{q>Q}\left(\sum_{p^{\prime} \leqslant q-2} \lambda_{p^{\prime}}\left\|w_{p^{\prime}}\right\|_{2} \lambda_{q-p^{\prime}}^{-\frac{3}{r}}\right)^{2} \\
& \lesssim c_{r} \nu \sum_{q>Q} \lambda_{q}^{2}\left\|w_{q}\right\|_{2}^{2}+c_{r} \nu \sum_{q>Q} \sum_{p^{\prime} \leqslant q-2} \lambda_{p^{\prime}}^{2}\left\|w_{p^{\prime}}\right\|_{2}^{2} \lambda_{q-p^{\prime}}^{-\frac{3}{r}} \\
& \lesssim c_{r} \nu \sum_{q>Q} \lambda_{q}^{2}\left\|w_{q}\right\|_{2}^{2}+c_{r} \nu \sum_{p^{\prime} \geqslant-1} \lambda_{p^{\prime}}^{2}\left\|w_{p^{\prime}}\right\|_{2}^{2} \sum_{q \geqslant p^{\prime}-2} \lambda_{q-p^{\prime}}^{-\frac{3}{r}} \\
& \lesssim c_{r} \nu\|\nabla w\|_{2}^{2} .
\end{aligned}
$$


The last term $I_{23}$ is similar to $I_{13}$ :

$$
\begin{aligned}
I_{23} & =\sum_{q>Q} \sum_{p \geqslant q-2}\left|\int_{\mathbb{T}^{3}} \Delta_{q}\left(u_{p} \otimes \tilde{w}_{p}\right) \nabla w_{q} d x\right| \\
& \leqslant \sum_{p>Q} \sum_{Q<q \leqslant p+2} \int_{\mathbb{T}^{3}}\left|\Delta_{q}\left(u_{p} \otimes \tilde{w}_{p}\right) \nabla w_{q}\right| d x+\sum_{Q<q \leqslant Q+2} \int_{\mathbb{T}^{3}}\left|\Delta_{q}\left(u_{Q} \otimes \tilde{w}_{Q}\right) \nabla w_{q}\right| d x \\
& \lesssim \sum_{p>Q}\left\|u_{p}\right\|\left\|_{r}\right\| \tilde{w}_{p}\left\|_{2} \sum_{Q<q \leqslant p+2} \lambda_{q}\right\| w_{q}\left\|_{\frac{2 r}{r-2}}+\lambda_{Q} \sum_{Q<q \leqslant Q+2}\right\| u_{Q}\|\infty\| \tilde{w}_{Q}\left\|_{2}\right\| w_{q} \|_{2} \\
& \lesssim c_{r} \nu \sum_{p>Q} \lambda_{p}^{1-\frac{3}{r}}\left\|\tilde{w}_{p}\right\|_{2} \sum_{Q<q \leqslant p+2} \lambda_{q}^{1+\frac{3}{r}}\left\|w_{q}\right\|_{2}+c_{r} \nu \lambda_{Q}^{2} \sum_{Q<q \leqslant Q+2}\left\|\tilde{w}_{Q}\right\|_{2}\left\|w_{q}\right\|_{2} \\
& \lesssim c_{r} \nu \sum_{p>Q} \lambda_{p}\left\|\tilde{w}_{p}\right\|_{2} \sum_{Q<q \leqslant p+2} \lambda_{q}\left\|w_{q}\right\|_{2} \lambda_{q-p}^{\frac{3}{r}}+c_{r} \nu \lambda_{Q}^{2} \sum_{Q<q \leqslant Q+2}\left\|w_{q}\right\|_{2}^{2} \\
& \lesssim c_{r} \nu\|\nabla w\|_{2}^{2} .
\end{aligned}
$$

Therefore, we have

$$
I_{2} \lesssim c_{r} \nu\|\nabla w\|_{2}^{2} .
$$

Combining (3.14) and (3.15), we conclude that there exists an adimensional constant $C>0$ that depends only on $r$, such that

$$
I_{1}+I_{2} \leqslant C c_{r} \nu\|\nabla w\|_{2}^{2} \text {. }
$$

Choosing $c_{r}:=1 /(2 C)$ we infer from (3.13) that

$$
\begin{aligned}
\|w(t)\|_{2}^{2} & \leqslant\left\|w\left(t_{0}\right)\right\|_{2}^{2}-\nu \int_{t_{0}}^{t}\|\nabla w\|_{2}^{2} d \tau \\
& \leqslant\left\|w\left(t_{0}\right)\right\|_{2}^{2}-\nu \kappa_{0}^{2} \int_{t_{0}}^{t}\|w\|_{2}^{2} d \tau
\end{aligned}
$$

where $\kappa_{0}=\frac{2 \pi}{L}$. Thus

$$
\|w(t)\|_{2}^{2} \leqslant\left\|w\left(t_{0}\right)\right\|_{2}^{2} e^{-\nu \kappa_{0}^{2}\left(t-t_{0}\right)}
$$

Taking the limit as $t \rightarrow \infty$ concludes the proof of Theorem 1.1, while taking the limit as $t_{0} \rightarrow-\infty$ concludes the proof of Theorem 1.2 .

\section{Explicit estimates of the AVERAGE DEtermining WAVENumber in TERMS OF KOLMOGOROV'S DISSIPATION WAVENUMBER}

In this section we show that the average determining wavenumber has a uniform upper bound. Recall that

$$
\Lambda_{u, r}(t):=\min \left\{\lambda_{q}: \lambda_{p}^{-1+\frac{3}{r}}\left\|u_{p}\right\|_{L^{r}}<c_{r} \nu, \forall p>q \text { and } \lambda_{q}^{-1}\left\|u_{\leqslant q}\right\|_{L^{\infty}}<c_{r} \nu, q \in \mathbb{N}\right\},
$$

where $r \in(2,3)$ and $c_{r}$ is an adimensional constant that depends only on $r$. To simplify the notations, denote $\Lambda:=\Lambda_{u, r}$ and let $Q$ be such that $\lambda_{Q}=\Lambda$.

We start with the following observation that will be used to estimate $\Lambda$.

Lemma 4.1. If $\lambda_{0}<\Lambda<\infty$, then

$$
\Lambda \leqslant\left(c_{r} \nu\right)^{-1} \max \left\{\Lambda^{\frac{3}{r}}\left\|u_{Q}\right\|_{r}, 2\left\|u_{\leqslant Q-1}\right\|_{r}\right\} .
$$

If $\Lambda=\infty$, then $\|u\|_{H^{\frac{1}{2}}}=\infty$. 
Proof. First consider the case where $\Lambda$ is finite. If both conditions in the definition of $\Lambda$ are satisfied for all $q \geqslant 0$, then $\Lambda=\lambda_{0}$. If $\Lambda>\lambda_{0}$, then both conditions in the definition of $\Lambda$ are satisfied for $q=Q$, but one of the conditions is not satisfied for $q=Q-1$, i.e.,

$$
\lambda_{Q}^{-1+\frac{3}{r}}\left\|u_{Q}\right\|_{r} \geqslant c_{r} \nu, \quad \text { or } \quad\left\|u_{\leqslant Q-1}\right\|_{\infty} \geqslant c_{r} \nu \lambda_{Q-1}=\frac{1}{2} c_{r} \nu \Lambda,
$$

and the conclusion of the lemma holds.

On the other hand, if $\Lambda=\infty$, then for every $q \in \mathbb{N}$ either

$$
\sup _{p>q} \lambda_{q}^{-1+\frac{3}{r}}\left\|u_{p}\right\|_{r} \geqslant c_{r} \nu, \quad \text { or } \quad \lambda_{q}^{-1}\left\|u_{\leqslant q}\right\|_{\infty} \geqslant c_{r} \nu .
$$

If

$$
\limsup _{p \rightarrow \infty} \lambda_{q}^{-1+\frac{3}{r}}\left\|u_{p}\right\|_{r} \geqslant c_{r} \nu
$$

then due to Bernstein's inequality,

$$
\|u\|_{H^{\frac{1}{2}}} \sim \sum_{q} \lambda_{q}\left\|u_{q}\right\|_{2}^{2} \gtrsim \sum_{q} \lambda_{q}^{-2+\frac{6}{r}}\left\|u_{q}\right\|_{r}^{2}=\infty
$$

and we are done. Otherwise, there exists $q^{* *}$ such that

$$
\lambda_{q}^{-1}\left\|u_{\leqslant q}\right\|_{\infty} \geqslant c_{r} \nu, \quad \forall q \geqslant q^{* *} .
$$

Now if $\|u\|_{H^{\frac{1}{2}}}<\infty$, then for any $\epsilon>0$ there exists $q^{*} \geqslant q^{* *}$ such that $\left\|u_{>q^{*}}\right\|_{H^{\frac{1}{2}}} \leqslant$ $\epsilon$. Then for $q \geqslant q^{*}$ we use Bernstein's inequality, Jensen's inequality, and (2.9) to obtain

$$
\begin{aligned}
\lambda_{q}^{-1}\left\|u_{\leqslant q}\right\|_{\infty} & \leqslant \sum_{p \leqslant q} \lambda_{q}^{-1} \lambda_{p}^{\frac{3}{2}}\left\|u_{p}\right\|_{2} \\
& \lesssim \sum_{p \leqslant q^{*} \leqslant q} \lambda_{q^{*}-q}^{\frac{1}{2}} \lambda_{p-q}^{\frac{1}{2}} \lambda_{p}^{\frac{1}{2}}\left\|u_{p}\right\|_{2}+\sum_{q^{*}<p \leqslant q} \lambda_{p-q} \lambda_{p}^{\frac{1}{2}}\left\|u_{p}\right\|_{2} \\
& \lesssim \lambda_{q^{*}-q}^{\frac{1}{2}}\left(\sum_{p \leqslant q^{*} \leqslant q} \lambda_{p-q}^{\frac{1}{2}} \lambda_{p}\left\|u_{p}\right\|_{2}^{2}\right)^{\frac{1}{2}}+\left(\sum_{q^{*}<p \leqslant q} \lambda_{p-q} \lambda_{p}\left\|u_{p}\right\|_{2}^{2}\right)^{\frac{1}{2}} \\
& \lesssim \lambda_{q^{*}-q}^{\frac{1}{2}}\left\|u_{\leqslant q^{*}}\right\|_{H^{\frac{1}{2}}}+\left\|u_{>q^{*}}\right\|_{H^{\frac{1}{2}}} \\
& \leqslant \lambda_{q^{*}-q}^{\frac{1}{2}}\left\|u_{\leqslant q^{*}}\right\|_{H^{\frac{1}{2}}}+\epsilon .
\end{aligned}
$$

Passing to the limit as $q \rightarrow \infty$ in the above inequality leads to

$$
c_{r} \nu \leqslant \epsilon,
$$

for arbitrarily small $\epsilon>0$, which is a contradiction. Thus we conclude that $\|u\|_{H^{\frac{1}{2}}}=$ $\infty$.

As the first consequence, we will show that $\Lambda(t)$ is locally integrable for every Leray-Hopf solution $u(t)$.

Lemma 4.2. The determining wavenumber $\Lambda$ enjoys the following bound:

$$
\Lambda-\lambda_{0} \lesssim \frac{\|\nabla u\|_{2}^{2}}{\nu^{2}} .
$$


Proof. If $\Lambda$ is infinite then $\|\nabla u\|_{2}$ is also infinite thanks to Lemma4.1. Now consider the case where $\lambda_{0}<\Lambda<\infty$. Then according to Lemma 4.1.

$$
\Lambda \leqslant\left(c_{r} \nu\right)^{-1} \Lambda^{\frac{3}{r}}\left\|u_{Q}\right\|_{r}, \quad \text { or } \quad \Lambda \leqslant 2\left(c_{r} \nu\right)^{-1}\left\|u_{\leqslant Q-1}\right\|_{\infty} .
$$

In the first case we use Bernstein's inequality to obtain

$$
\begin{aligned}
\Lambda & \leqslant\left(c_{r} \nu\right)^{-2} \Lambda^{\frac{6}{r}-1}\left\|u_{Q}\right\|_{r}^{2} \\
& \lesssim\left(c_{r} \nu\right)^{-2} \Lambda^{2}\left\|u_{Q}\right\|_{2}^{2} \\
& \lesssim\left(c_{r} \nu\right)^{-2}\|\nabla u\|_{2}^{2} .
\end{aligned}
$$

In the second case,

$$
\begin{aligned}
\Lambda & \leqslant 4\left(c_{r} \nu\right)^{-2} \Lambda^{-1}\left\|u_{\leqslant Q-1}\right\|_{\infty}^{2} \\
& \lesssim\left(c_{r} \nu\right)^{-2} \Lambda^{-1}\left(\sum_{q<Q}\left\|u_{q}\right\|_{\infty}\right)^{2} \\
& \lesssim\left(c_{r} \nu\right)^{-2} \Lambda^{-1}\left(\sum_{q<Q} \lambda_{q}^{\frac{3}{2}}\left\|u_{q}\right\|_{2}\right)^{2} \\
& =\left(c_{r} \nu\right)^{-2}\left(\sum_{q<Q} \lambda_{q}\left\|u_{q}\right\|_{2} 2^{\frac{1}{2}(q-Q)}\right)^{2} \\
& \lesssim\left(c_{r} \nu\right)^{-2} \sum_{q<Q} \lambda_{q}^{2}\left\|u_{q}\right\|_{2}^{2} 2^{\frac{1}{2}(q-Q)} \\
& \lesssim\left(c_{r} \nu\right)^{-2}\|\nabla u\|_{2}^{2},
\end{aligned}
$$

and the conclusion of the lemma holds again.

We can now compare the average determining wavenumber with Kolmogorov's dissipation wavenumber, often defined as

$$
\kappa_{\mathrm{d}}:=\left(\frac{\varepsilon}{\nu^{3}}\right)^{\frac{1}{4}},
$$

where $\varepsilon$ is the average energy dissipation rate

$$
\varepsilon:=\frac{\nu}{L^{3}}\left\langle\|\nabla u\|_{2}^{2}\right\rangle=\frac{\nu}{T L^{3}} \int_{t}^{t+T}\|\nabla u(\tau)\|_{2}^{2} d \tau,
$$

and $\langle\cdot\rangle$ denotes the time average. Then Lemma 4.2 yields the following bound:

$$
\langle\Lambda\rangle-\lambda_{0} \lesssim \frac{\left\langle\|\nabla u\|_{2}^{2}\right\rangle}{\nu^{2}}=\frac{\varepsilon}{\nu^{3}} L^{3}=\kappa_{\mathrm{d}}^{4} \lambda_{0}^{-3}
$$

However, in this argument we used Bernstein's inequalities that might not be sharp in a turbulent regime. The level of saturation of Bernstein's inequalities is measured by a parameter $d$, called the intermittency dimension (see [7] where the notions of active regions, eddies, and intermittency are defined mathematically). The number of eddies at scale $\ell$ grows as

$$
\text { Number of eddies } \sim\left(\frac{L}{\ell}\right)^{d} \text {. }
$$


The case $d=3$ corresponds to Kolmogorov's regime where eddies occupy the whole region for each scale in the inertial range. The other extreme case is $d=0$, where the number of eddies is of order one on all the scales, in which case Bernstein's inequalities are sharp. A recent DNS performed by Kaneda et al. 24] on the Earth Simulator suggests that $d \approx 2.7$. The presence of intermittency requires the following modification of the classical definition (4.16):

$$
\kappa_{\mathrm{d}}:=\left(\frac{\varepsilon}{\nu^{3}}\right)^{\frac{1}{d+1}}, \quad \varepsilon:=\frac{\nu}{L^{d}}\left\langle\|\nabla u\|_{2}^{2}\right\rangle,
$$

where $d \in[0,3]$ is the intermittency dimension. This parameter is chosen so that

$$
\left\langle\sum_{q \leqslant Q} \lambda_{q}^{-1+\frac{6}{r}+d\left(1-\frac{2}{r}\right)}\left\|u_{q}\right\|_{r}^{2}\right\rangle \lesssim \lambda_{0}^{d\left(1-\frac{2}{r}\right)}\left\langle\sum_{q \leqslant Q} \lambda_{q}^{2}\left\|u_{q}\right\|_{2}^{2}\right\rangle .
$$

This can be done since by Bernstein's inequality

$$
\lambda_{0}^{3-\frac{6}{r}} \lambda_{q}^{-1+\frac{6}{r}}\left\|u_{q}\right\|_{2}^{2} \lesssim \lambda_{q}^{-1+\frac{6}{r}}\left\|u_{q}\right\|_{r}^{2} \lesssim \lambda_{q}^{2}\left\|u_{q}\right\|_{2}^{2} .
$$

Note that $-1+d\left(1-\frac{2}{r}\right)<0$ since $d \leqslant 3$ and $r<3$. Therefore,

$$
\begin{aligned}
\Lambda^{-1+d\left(1-\frac{2}{r}\right)}\left\|u_{\leqslant Q-1}\right\|_{\infty}^{2} & \lesssim \Lambda^{-1+d\left(1-\frac{2}{r}\right)}\left(\sum_{q<Q}\left\|u_{q}\right\|_{\infty}\right)^{2} \\
& \lesssim \Lambda^{-1+d\left(1-\frac{2}{r}\right)}\left(\sum_{q<Q} \lambda_{q}^{\frac{3}{r}}\left\|u_{q}\right\|_{r}\right)^{2} \\
& =\left(\sum_{q<Q} \lambda_{q}^{-\frac{1}{2}+\frac{3}{r}+\frac{d}{2}\left(1-\frac{2}{r}\right)}\left\|u_{q}\right\|_{r}\left(L \lambda_{Q-q}\right)^{-\frac{1}{2}+\frac{d}{2}\left(1-\frac{2}{r}\right)}\right)^{2} \\
& \lesssim_{r} \sum_{q<Q} \lambda_{q}^{-1+\frac{6}{r}+d\left(1-\frac{2}{r}\right)}\left\|u_{q}\right\|_{r}^{2} .
\end{aligned}
$$

Now, thanks to Lemma 4.1, we have

$$
\Lambda^{-1+\frac{6}{r}+d\left(1-\frac{2}{r}\right)}\left\|u_{Q}\right\|_{r}^{2} \geqslant\left(c_{r} \nu\right)^{2} \Lambda^{1+d\left(1-\frac{2}{r}\right)}
$$

or

$$
4 \Lambda^{-1+d\left(1-\frac{2}{r}\right)}\left\|u_{\leqslant Q-1}\right\|_{\infty}^{2} \geqslant\left(c_{r} \nu\right)^{2} \Lambda^{1+d\left(1-\frac{2}{r}\right)},
$$

provided $\lambda_{0}<\Lambda<\infty$. Therefore,

$$
\left(c_{r} \nu\right)^{2} \Lambda^{1+d\left(1-\frac{2}{r}\right)} \leqslant \Lambda^{-1+\frac{6}{r}+d\left(1-\frac{2}{r}\right)}\left\|u_{Q}\right\|_{r}^{2}+4 \Lambda^{-1+d\left(1-\frac{2}{r}\right)}\left\|u_{\leqslant Q-1}\right\|_{\infty}^{2},
$$

whenever $\lambda_{0}<\Lambda<\infty$. Combining this with (4.20) we obtain

$$
\begin{aligned}
\left(c_{r} \nu\right)^{2} \Lambda^{1+d\left(1-\frac{2}{r}\right)} & \varliminf_{r} \Lambda^{-1+\frac{6}{r}+d\left(1-\frac{2}{r}\right)}\left\|u_{Q}\right\|_{r}^{2}+\sum_{q<Q} \lambda_{q}^{-1+\frac{6}{r}+d\left(1-\frac{2}{r}\right)}\left\|u_{q}\right\|_{r}^{2} \\
& =\sum_{q \leqslant Q} \lambda_{q}^{-1+\frac{6}{r}+d\left(1-\frac{2}{r}\right)}\left\|u_{q}\right\|_{r}^{2} .
\end{aligned}
$$

Note that when $\Lambda=\infty$ the bound (4.21) holds as well since both sides in these inequalities are infinite due to Lemma 4.1. Taking the time average of (4.21) and 
using (4.19), we arrive at

$$
\begin{aligned}
\left(c_{r} \nu\right)^{2}\left\langle\Lambda^{1+d\left(1-\frac{2}{r}\right)}\right\rangle_{>\lambda_{0}} & \lesssim r\left\langle\sum_{q \leqslant Q} \lambda_{q}^{-1+\frac{6}{r}+d\left(1-\frac{2}{r}\right)}\left\|u_{q}\right\|_{r}^{2}\right\rangle_{>\lambda_{0}} \\
& \lesssim \lambda_{0}^{d\left(1-\frac{2}{r}\right)}\left\langle\sum_{q \leqslant Q} \lambda_{q}^{2}\left\|u_{q}\right\|_{2}^{2}\right\rangle_{>\lambda_{0}} \\
& \lesssim \lambda_{0}^{d\left(1-\frac{2}{r}\right)}\left\langle\|\nabla u\|_{2}^{2}\right\rangle \\
& =: \lambda_{0}^{-\frac{2 d}{r}} \frac{\varepsilon}{\nu},
\end{aligned}
$$

where $\langle g\rangle_{>\lambda_{0}}=\left\langle 1_{g>\lambda_{0}} g\right\rangle$. Finally, using Jensen's inequality,

$$
\begin{aligned}
\langle\Lambda\rangle-\lambda_{0}=\langle\Lambda\rangle_{>\lambda_{o}} & =\left(\frac{\left(c_{r} \nu\right)^{2}\langle\Lambda\rangle_{>\lambda_{0}}^{1+d\left(1-\frac{2}{r}\right)}}{\left(c_{r} \nu\right)^{2}}\right)^{\frac{1}{1+d\left(1-\frac{2}{r}\right)}} \\
& \lesssim\left(\frac{\left(c_{r} \nu\right)^{2}\left\langle\Lambda^{1+d\left(1-\frac{2}{r}\right)}\right\rangle_{>\lambda_{0}}}{\left(c_{r} \nu\right)^{2}}\right)^{\frac{1}{1+d\left(1-\frac{2}{r}\right)}} \\
& \lesssim_{r}\left(\frac{\varepsilon}{\nu^{3}}\right)^{\frac{1}{1+d\left(1-\frac{2}{r}\right)}} \lambda_{0}^{-\frac{2 d}{r+d(r-2)}}
\end{aligned}
$$

Comparing it with Kolmogorov's dissipation wavenumber (4.18), we get

$$
\langle\Lambda\rangle-\lambda_{0} \lesssim_{r} \kappa_{\mathrm{d}}^{\frac{1+d}{1+d\left(1-\frac{2}{r}\right)}} \lambda_{0}^{-\frac{2 d}{r+d(r-2)}} .
$$

In case of extreme intermittency $d=0$ the powers do not depend on $r$, so we can just choose $r=5 / 2$ inferring that the average determining wavenumber is bounded by Kolmogorov's dissipation wavenumber $\kappa_{d}$ :

$$
\langle\Lambda\rangle-\lambda_{0} \lesssim \frac{\varepsilon}{\nu^{3}}=\kappa_{\mathrm{d}}, \quad \text { when } \quad d=0 .
$$

However, in Kolmogorov's regime $d=3$, the bound becomes $\kappa_{\mathrm{d}}^{2+}$ since $r$ can only be taken less than 3 . More precisely, for any $d \leqslant 3$ we have the following bound:

$$
\langle\Lambda\rangle-\lambda_{0} \lesssim_{r} \kappa_{\mathrm{d}}^{\frac{2 r}{2 r-3}} \lambda_{0}^{-\frac{3}{2 r-3}}=\kappa_{\mathrm{d}}^{2+\delta} \lambda_{0}^{-1-\delta},
$$

where $\delta=\frac{6-2 r}{2 r-3}$ can be arbitrarily small when $r$ is chosen close to 3 .

\section{EXPLICIT ESTIMATES OF THE AVERAGE DETERMINING WAVENUMBER IN TERMS OF THE GRASHOF NUMBER}

It is well known that the 3D Navier-Stokes equation possesses an absorbing ball in $L^{2}$

$$
B:=\left\{u \in L^{2}\left(\mathbb{T}^{3}\right):\|u\|_{2} \leqslant R\right\},
$$

where $R$ is any number larger than $\frac{\|f\|_{H^{-1}}}{\nu \kappa_{0}}$ and $\kappa_{0}=2 \pi \lambda_{0}=2 \pi / L$, which can be expressed in terms of the adimensional Grashof number

$$
G:=\frac{\|f\|_{H^{-1}}}{\nu^{2} \kappa_{0}^{1 / 2}}
$$


as $R>\nu \kappa_{0}^{-1 / 2} G$. Then for any Leray solution $u(t)$ there exists $t_{0}$, depending only on $\|u(0)\|_{2}$, such that

$$
u(t) \in B \quad \forall t>t_{0} .
$$

Then the evolutionary system consisting of Leray-Hopf solutions in the absorbing ball posseses a weak global attractor $\mathcal{A}$, which has the following structure [17, 13]:

$$
\mathcal{A}=\{u(0): u(\cdot) \text { is a complete bounded Leray-Hopf solution to the NSE }\} \text {. }
$$

The set $\mathcal{A} \subset B$ is the minimal $L^{2}$ weakly closed weakly attracting set, it is $L^{2}$-weak omega limit of $B$ (see [2, 9]), and $\|u\|_{2} \leqslant \nu \kappa_{0}^{-1 / 2} G$ for all $u \in \mathcal{A}$. See also [16] for topological properties of $\mathcal{A}$.

Let $u(t)$ be a trajectory on the global attractor $\mathcal{A}$. To bound the average determining wavenumber in terms of the Grashof number we use the energy inequality:

$$
\begin{aligned}
0 \leqslant\left\|u\left(t_{0}+t\right)\right\|_{2}^{2} & \leqslant \limsup _{t \rightarrow t_{0}+}\|u(t)\|_{2}^{2}-2 \nu \int_{t_{0}}^{t_{0}+T}\|\nabla u(t)\|_{2}^{2} d t+2 \int_{t_{0}}^{t_{0}+T}(f, u) d t \\
& \leqslant \nu^{2} \kappa_{0}^{-1} G^{2}-\nu \int_{t_{0}}^{t_{0}+T}\|\nabla u(t)\|_{2}^{2} d t+\frac{1}{\nu} \int_{t_{0}}^{t_{0}+T}\|f\|_{H^{-1}}^{2} d t .
\end{aligned}
$$

Therefore

$$
\begin{aligned}
\frac{1}{T} \int_{t_{0}}^{t_{0}+T}\|\nabla u(t)\|_{2}^{2} d t & \leqslant \frac{\nu G^{2}}{T \kappa_{0}}+\frac{\|f\|_{H^{-1}}^{2}}{\nu^{2}} \\
& \leqslant \frac{\nu G^{2}}{T \kappa_{0}}+\kappa_{0} \nu^{2} G^{2}
\end{aligned}
$$

Then (4.17) imply

$$
\langle\Lambda\rangle-\lambda_{0} \lesssim \frac{\left\langle\|\nabla u\|_{2}^{2}\right\rangle}{\nu^{2}} \leqslant \frac{G^{2}}{\nu T \kappa_{0}}+\kappa_{0} G^{2} .
$$

To take into account intermittency, we can use (4.22) instead of (4.17) to obtain

$$
\begin{aligned}
\langle\Lambda\rangle-\lambda_{0} & \lesssim_{r}\left(\frac{\varepsilon}{\nu^{3}}\right)^{\frac{1}{1+d\left(1-\frac{2}{r}\right)}} \lambda_{0}^{-\frac{2 d}{r+d(r-2)}} \\
& =\left(\frac{\lambda_{0}^{d\left(1-\frac{2}{r}\right)}\left\langle\|\nabla u\|_{2}^{2}\right\rangle}{\nu^{2}}\right)^{\frac{1}{1+d\left(1-\frac{2}{r}\right)}} \\
& \leqslant \kappa_{0}\left(\frac{G^{2}}{\nu T \kappa_{0}^{2}}+G^{2}\right)^{\frac{1}{1+d\left(1-\frac{2}{r}\right)}}
\end{aligned}
$$

where $d \in[0,3)$ is the intermittency parameter from Section 4 . In the case of extreme intermittency $d=0$ this bound is the same as (5.23), proportional to $G^{2}$. However, in Kolmogorov's regime where $d=3$, the average determining number is bounded by $G^{1+}$. More precisely,

$$
\langle\Lambda\rangle-\lambda_{0} \lesssim_{\delta} \kappa_{0}\left(\frac{G^{2}}{\nu T \kappa_{0}^{2}}+G^{2}\right)^{\frac{1}{2}+\delta}, \quad d=3,
$$

where $\delta=\frac{3-r}{4 r-6} \rightarrow 0$ as $r \rightarrow 3-$. 


\section{The CASE OF ENERgy EQUALITY}

In this section we consider the case where one of the solutions of the 3D NavierStokes equations satisfies the energy equality, e.g., a steady state or a solution belonging to Onsager's space $L^{3}\left(0, \infty ; B_{3, \infty}^{1 / 3}\right)$.

Theorem 6.1. Let $v(t)$ be a weak solution of the $3 D$ NSE satisfying the energy equality, and $Q(t)$ be such that $\Lambda_{v, r}(t)=\lambda_{Q(t)}$ for some $r \in(2,3)$. If $u(t)$ is a Leray-Hopf weak solution such that

$$
u(t)_{\leqslant Q(t)}=v(t)_{\leqslant Q(t)}, \quad \forall t>0,
$$

then

$$
\lim _{t \rightarrow \infty}\|u(t)-v(t)\|_{L^{2}}=0
$$

Proof. We know that $v$ satisfies energy equality

$$
\int_{\mathbb{T}^{3}} \frac{1}{2} v^{2} d x+\nu \int_{0}^{t} \int_{\mathbb{T}^{3}}|\nabla v|^{2} d x d \tau=\int_{\mathbb{T}^{3}} \frac{1}{2} v_{0}^{2} d x+\int_{0}^{t} \int_{\mathbb{T}^{3}} f v d x d \tau ;
$$

and $u$ satisfies energy inequality

$$
\int_{\mathbb{T}^{3}} \frac{1}{2} u^{2} d x+\nu \int_{0}^{t} \int_{\mathbb{T}^{3}}|\nabla u|^{2} d x d \tau \leqslant \int_{\mathbb{T}^{3}} \frac{1}{2} u_{0}^{2} d x+\int_{0}^{t} \int_{\mathbb{T}^{3}} f u d x d \tau ;
$$

Computing the energy of the difference $w:=u-v$

$$
\begin{aligned}
& \int_{\mathbb{T}^{3}} \frac{1}{2}|w|^{2} d x+\nu \int_{0}^{t} \int_{\mathbb{T}^{3}}|\nabla w|^{2} d x d \tau \\
= & \int_{\mathbb{T}^{3}} \frac{1}{2} u^{2} d x+\int_{\mathbb{T}^{3}} \frac{1}{2} v^{2} d x+\nu \int_{0}^{t} \int_{\mathbb{T}^{3}}|\nabla u|^{2} d x d \tau+\nu \int_{0}^{t} \int_{\mathbb{T}^{3}}|\nabla v|^{2} d x d \tau \\
& -\int_{\mathbb{T}^{3}} u v d x-2 \nu \int_{0}^{t} \int_{\mathbb{T}^{3}} \nabla u \nabla v d x d \tau,
\end{aligned}
$$

combining (6.25) and (6.26) we conclude

$$
\begin{aligned}
& \int_{\mathbb{T}^{3}} \frac{1}{2}|w|^{2} d x+\nu \int_{0}^{t} \int_{\mathbb{T}^{3}}|\nabla w|^{2} d x d \tau \\
\leqslant & \int_{\mathbb{T}^{3}} \frac{1}{2}\left(u_{0}^{2}+v_{0}^{2}\right) d x+\int_{0}^{t} \int_{\mathbb{T}^{3}} f(u+v) d x d \tau \\
& -\int_{\mathbb{T}^{3}} u v d x-2 \nu \int_{0}^{t} \int_{\mathbb{T}^{3}} \nabla u \nabla v d x d \tau .
\end{aligned}
$$

As for the $L^{2}$ inner product of $u$ and $v$, we have (see, e.g., [29])

$$
\begin{aligned}
& \int_{\mathbb{T}^{3}} u_{0} v_{0} d x-\int_{\mathbb{T}^{3}} u v d x-2 \nu \int_{0}^{t} \int_{\mathbb{T}^{3}} \nabla u \nabla v d x d \tau \\
= & -\int_{0}^{t} \int_{\mathbb{T}^{3}} w \cdot \nabla v w d x d \tau-\int_{0}^{t} \int_{\mathbb{T}^{3}} f(u+v) d x d \tau .
\end{aligned}
$$

Combining (6.27) and (6.28) yields

$$
\int_{\mathbb{T}^{3}} \frac{1}{2}|w|^{2} d x+\nu \int_{0}^{t} \int_{\mathbb{T}^{3}}|\nabla w|^{2} d x d \tau \leqslant \int_{\mathbb{T}^{3}} \frac{1}{2}|w(0)|^{2} d x+\int_{0}^{t} \int_{\mathbb{T}^{3}}|w \cdot \nabla v w| d x d \tau .
$$


It then follows from estimate (3.14) that for a small constant $c_{r}$

$$
\int_{\mathbb{T}^{3}}|w|^{2} d x+\nu \int_{0}^{t} \int_{\mathbb{T}^{3}}|\nabla w|^{2} d x d \tau \leqslant \int_{\mathbb{T}^{3}}|w(0)|^{2} d x
$$

Applying Poincaré's inequality, we have

$$
\int_{\mathbb{T}^{3}}|w|^{2} d x \leqslant \int_{\mathbb{T}^{3}}|w(0)|^{2} d x-\kappa_{0} \nu \int_{0}^{t} \int_{\mathbb{T}^{3}}|w|^{2} d x d \tau
$$

Thus

$$
\|w(t)\|_{2}^{2} \leqslant\|w(0)\|_{2}^{2} e^{-\kappa_{0} \nu t}
$$

\section{ACKNOWLEDGMENT}

The authors thank the anonymous referee for careful reading the manuscript and constructive comments.

\section{REFERENCES}

[1] H. Bahouri, J. Chemin, and R. Danchin. Fourier analysis and nonlinear partial differential equations. Grundlehrender Mathematischen Wissenschaften, 343. Springer, Heidelberg, 2011.

[2] A. Cheskidov. Global attractors of evolutionary systems. Journal of Dynamics and Differential Equations, 21: 249-268, 2009.

[3] A. Cheskidov, P. Constantin, S. Friedlander and R. Shvydkoy. Energy conservation and Onsager's conjecture for the Euler equations. Nonlinearity, 21: 1233-1252, 2008.

[4] A. Cheskidov and M. Dai. Determining modes for the surface Quasi-Geostrophic equation. arXiv:1507.01075 2015.

[5] A. Cheskidov and C. Foias. On global attractors of the 3D Navier-Stokes equations. J. Differential Equations, Vol. 231, (2): 714-754, 2006.

[6] A. Cheskidov and R. Shvydkoy. A unified approach to regularity problems for the $3 D$ NavierStokes and Euler equations: the use of Kolmogorov's dissipation range. J. Math. Fluid Mech., 16:263-273, 2014.

[7] A. Cheskidov and R. Shvydkoy. Euler Equations and Turbulence: Analytical Approach to Intermittency. SIAM J. Math. Anal., 46(1), 353-374, 2014.

[8] B. Cockburn, D. Jones, and E. Titi. Estimating the number of asymptotic degrees of freedom for nonlinear dissipative systems. Mathematics of Computation, Vol. 66 (219): 1073-1087, 1997.

[9] P. Constantin and C. Foias. Navier-Stokes Equations. University of Chicago press.

[10] P. Constantin, C. Foias, O. P. Manley, and R. Temam. Determining modes and fractal dimension of turbulent flows. J. Fluid Mech., 150:427-440, 1985.

[11] P. Constantin, C. Foias, and R. Temam. On the dimension of the attractors in twodimensional turbulence. Physica D, 30, 284-296, 1988.

[12] C. Foias, M. Jolly, R. Kravchenko and E. Titi. A determining form for the 2D Navier-Stokes equations - the Fourier modes case. J. Math. Phys., 53(11), 115623, 30 pp, 2012.

[13] C. Foias, O. Manley, R. Rosa, and R. Temam. Navier-Stokes equations and turbulence. Vol. 83 of Encyclopedia of Mathematics and its Applications. Cambridge University Press, Cambridge, 2001.

[14] C. Foias, O. P. Manley, R Temam, and Y. M. Tréve. Asymptotic analysis of the NavierStokes equations. Phys. D, 9(1-2), 157-188, 1983.

[15] C. Foias and G. Prodi. Sur le comportement global des solutions non-stationnaires des équations de Navier-Stokes en dimension 2. Rend. Sem. Mat. Univ. Padova 39:1-34, 1967.

[16] C. Foias, R. Rosa, and R. Temam. Topological properties of the weak global attractor of the three-dimensional Navier-Stokes equations. Discrete and Continuous Dynamical Systems, Vol. (4): 1611-1631, 2010.

[17] C. Foias and R. Temam. Some analytic and geometric properties of the solutions of the Navier-Stokes equations. J. Math. Pures Appl., 58, 339-368, 1979. 
[18] C. Foias and R. Temam. Determination of the solutions of the Navier-Stokes equations by a set of nodal values. Math. Comput., 43, 117-133, 1984.

[19] C. Foias and R. Temam. The connection between the Navier-Stokes equations, and turbulence theory. Directions in Partial Differential Equations, Publ. Math. Res. Center Univ. Wisconsin, 55-73, 1985.

[20] C. Foias and E. S. Titi. Determining nodes, finite difference schemes and inertial manifolds. Nonlinearity, 135-153, 1991.

[21] U. Frisch. Turbulence: The legacy of A. N. Kolmogorov. Cambridge University Press, Cambridge, 1995.

[22] L. Grafakos. Modern Fourier Analysis. Second edition. Graduate Texts in Mathematics, 250. Springer, New York, 2009.

[23] D. A. Jones and E. S. Titi. Upper bounds on the number of determining modes, nodes, and volume elements for the Navier-Stokes equations. Indiana Univ. Math. J. 42(3):875-887, 1993.

[24] Y. Kaneda, T. Ishihara, M. Yokokawa, K. Itakura, and A. Uno. Energy dissipation rate and energy spectrum in high resolution direct numerical simulations of turbulence in a periodic box Physics of Fluids, 15(2):21-24, 2003.

[25] A. N. Kolmogorov. The local structure of turbulence in incompressible viscous fluids at very large Reynolds numbers. Dokl. Akad. Nauk. SSSR 30: 301?305, 1941.

[26] J. Leray. Sur le mouvement dun liquide visqueux emplissant lespace. Acta Math., 63(1):193248, 1934.

[27] J. C. Robinson, J. L. Rodrigo, and W. Sadowski. The Three-Dimensional Navier-Stokes Equations. Cambridge University Press, 2016.

[28] G. R. Sell. Global attractors for the three-dimensional Navier-Stokes equations. Journal of Dynamics and Differential Equations, Vol. 8, Issue 1: 1-33, 1996.

[29] R. Temam. Navier-Stokes Equations: Theory and Numerical Analysis. AMS Chelsea, Providence, Rhode Island, 2000.

Department of Mathematics, Stat. and Comp.Sci., University of Illinois Chicago, ChicAgo, IL 60607,USA

Email address: acheskid@uic.edu

Department of Applied Mathematics, Stat. and Comp.Sci., University of Illinois Chicago, Chicago, IL 60607,USA

Email address: mdai@uic.edu

Department of Mathematics, Stat. and Comp.Sci., University of Illinois Chicago, ChicAgo, IL 60607,USA

Email address: lkavli2@uic.edu 